\title{
Application of Wavelet Transform in MCG-signal Denoising
}

\author{
Yucai Dong, Hongtao Shi, Junzhi Luo, Gehua Fan \& Caiping Zhang \\ Institute of Nonlinear Science, Academy of Armored Force Engineering, Beijing 100072, China
}

\begin{abstract}
In this paper, the principle of denoising with wavelet transform are discussed. The application of wavelet threshold denoising method in MCG-signal processing problem is introduced. Program is written by MATLAB to realize wavelet threshold denoising method. The results show that the wavelet threshold denoising method in the MCG-signal denoising can not only restrain the noise effectively, but also reserve the fault character information in the original signal. The signals by this method are better improvements than traditional method.
\end{abstract}

Keywords: MCG-signal, Wavelet Transform, Signal Denoising

\section{Introduction}

With the continuous development of biomagnetism detection technology, the method of Magneto Cardio Graphy has been applied to the stage of clinical application with the advantages of non-traumatic, non-contact, highly sensitive, non-radiative and so on. The Magneto Cardio Graphy, using SQUID as the detecting devices to detect and analyze the MCG-signal, is a highly sensitive detecting equipment which detect and diagnose the heart disease. How to denoise is the key to detect MCG-signal because the MCG-signal of human beings is very faint which is only one part per million of geomagnetic field.(Chen, Lin. 2006) (zhang, xiyuan. 2004) analyze and research noise cancellation about the MCG-signal making use of fourier transform method, which remove the effect of noises to some extent. (Hu, Rong. 2005) applied the mathematical morphological filtering method to analyze and process the MCG-signal and the resulting tests show that this method can eliminate random and high frequency noise effectively, but the disadvantage is the selection of structural elements affects the resulting test very much. (Wang, wei. 2008) applied the morphological filtering method to construct two kinds of structural elements, which can preserve the component of $\mathrm{T}$ wave in the MCG-signal and also remove the baseline drift of the MCG-signal. This paper makes use of wavelet transform to analyze and process noise cancellation about the MCG-signal in the view of mathematics. The resulting tests reveals that this method is effective which may not only remove random and high frequency noise but also preserve the signal.

\section{The principle of wavelet transformation}

The wavelet transform is an analyzing method having good locally features in both time domain and frequency domain. The wavelet transform adopts gradually precise spatial domain step to process high frequency, so it can focus on optional details of the analyzed target, which affords a new processing method for the signal process. However, the traditional fourier transform which completely analyzes signals in the frequency domain has no resolution for the signals in the time domain.(Jia, Mingtao \& Wang, Yongchen. 2003)

The concept of the wavelet transform is: the basic wavelet function $\varphi$ is translated and expanded to obtain one function family. (Li, Bicheng \& Luo, Jianshu. 2005)

$\varphi_{a, b}(x)=|a|^{-1 / 2} \varphi\left(\frac{x-b}{a}\right), \quad(a, b \in R, a \neq 0)$

Where $a$ is the expansion factor and $b$ is the translation factor. Analyze the signal through making inner product with the function family and the signal. The wavelet function should satisfy two conditions: (1) $\varphi$ is continual and attenuation; (2) the integral of $\varphi$ is zero.

When the wavelet function is determined, the continual wavelet transform and inverse transform of the signal $f(t)$ are:

$W_{f}(a, b)=|a|^{-1 / 2} \int_{R} f(t) \overline{\varphi\left(\frac{t-b}{a}\right)} d t$

$f(t)=\frac{1}{C_{\varphi}} \int_{R} \int_{R} \frac{1}{a^{2}} \varphi\left(\frac{t-b}{a}\right) W_{f}(a, b) d a d b$ 
where $C_{\varphi}=\int_{R} \frac{|\hat{\varphi}(\omega)|^{2}}{\omega} d \omega, \overline{\varphi(t)}$ denotes the complex conjugate of $\varphi(t)$ and $\hat{\varphi}$ denotes the fourier transformation of $\varphi$.

In the practical application, we commonly adopt discrete wavelet transform and the definition is:

$D_{f}(j, k)=2^{j / 2} \int_{R} f(t) \overline{\varphi\left(2^{j} t-k\right)} d t$

Where $\mathrm{j}, \mathrm{k}$ are the discrete numbers of the expansion factor $a$ and the translation factor $b$, separately.

\section{The application of wavelet transform in signal denoising}

Generally speaking all the practical signals are signals with noise, for example, all kinds of physiological electrical signals including cardiac electricity, brain electricity and gastric electricity in the biomedicine and some other non-physiological electrical signals (as pulse wave, MCG et al.) which are transformed to electrical signals are submerged in the strong noises resulting in losing the values of medical diagnosis. So noise cancellation and filtering are the important content of processing biomedical signals. The traditional method is that one separates the signals and noises according to the different frequency bands of signal and noise, which adopts the fourier transformation to transform the time domain to the frequency domain. When the frequency bands of signals and noises overlap, the effects of this method will decrease apparently and the contradiction between protecting the local feature of signals and restraining noises exists. The wavelet transform has good quality of time-frequency localization, which afford powerful tool for solving this problem. Now, the wavelet technique is widely studied in the signal denoising and obtains very good application effect. The familiar basic methods of wavelet denoising are: module maximum of wavelet transform denoising, noise cancellation based the scale coherency of wavelet transform and wavelet threshold denoising.

\section{1 module maximum of wavelet transform denoising}

The module maximums of signals and noises in the separate scale of wavelet transform have completely different traveling characteristics. The Lipschitz exponents of common signals are usually more than zero and the number of module maximums is identical in smaller scale. However, the Lipschitz exponents of noises are usually less than zero. For example, the white Gaussian noise is distributing randomly with almost singular everywhere, which has negative Lipschitz exponents. One can observe the gradually changing law of module maximums of wavelet transform and the distributing law of module maximums in different scales to estimate the location of singular points and the corresponding Lipschitz exponents, which can separate signals and noises to realize wavelet denoising. This method depends on the noises little, which does not need the variance of noises, so it suits signal denoising with low S/N. However, the calculation accuracy is low and the effects of noise cancellation can not be satisfied.

\section{2 noise cancellation based the scale coherency of wavelet transform}

The different spreading characteristics of the wavelet transforms of signals and noises in different scales show that the wavelet transform of signals has strong coherency between different scales and the coherency in margin is very strong, however, the wavelet transform of noises does not have the obvious coherency in different scales and the wavelet transform of noises mainly focus on different levels of small scales. According to different characteristics of wavelet transforms of signals and noises in different scales, one proposes the method making use of the coherency of wavelet transform to distinguish signals and noises. This method can obtain very good denoising effects and the effects are stable, which suits for the signals with high S/N. The disadvantages are large amount of calculation and the requirement to evaluate the variance of noises.

\subsection{Wavelet threshold denoising method}

The main theory of wavelet threshold denoising method is: the wavelet transform, especially the orthogonal wavelet transform, has strong denoising coherency, which can focus the energy of signals included in the wavelet domain on some big wavelet coefficients and distribute the energy of noises to all the wavelet domain. Therefore, the amplitude of wavelet coefficient of signals is bigger than that of noises, so it can be thought that the wavelet coefficients with bigger amplitude are mainly signals and the wavelet coefficients with smaller amplitude are noises to large extent. Therefore, the coefficients of signals are reserved and most of coefficients of noises are reduced to zero using the threshold method.

The wavelet threshold denoising method mainly suits for signals mixed with white noises. The advantages are highly calculating velocity, whose calculation complexity is $O(N)$ where $N$ is the length of signal, and the 
noises are restrained almost and the characteristic plug points reflecting the original signals can be reserved. The disadvantages are the denoising effects depend on $\mathrm{S} / \mathrm{N}$ and the choice of threshold has very important influences on the denoising effects. There are many methods of choosing thresholds, and the most common method is VisuShrink, which adopts the global uniform threshold $\sigma \sqrt{2 \log N}$, where $\sigma$ is the standard deviation of noises and $N$ is the length of signals.

\section{Noise cancellation on MCG-signal applying wavelet threshold denoising}

The MCG-signals are surveyed in the shielding room using high-tc squids. The data are sampled with the frequency of 1000 and a total recording length of 5000. The original data are shown as figure 1.

Considering the frequencies of MCG-signals mainly concentrate below 50HZ and the MCG-signals are very faint relative to power line interference. So the data should be preprocessed with low-pass filter to eliminate power line interference and high frequency noises interference, which are shown as figure 2. From the figure, it is found that the MCG-signals include a large amount of random noises interference after the traditional low-pass filter processing and these signal data have no practical meaning for the clinical diagnosing, from which the random interferences have to be eliminated for the future analytical processing.

The processed signals are denoised by applying wavelet threshold denoising. The idiographic stages are:

(1) The signals are decomposed by wavelet in all the scales and all the wavelet coefficients in the condition of low resolution and large scales are preserved. In the process of decomposition, choosing the appropriate wavelet and reasonable decomposing layers are very important for the denoising results. Figure 3 chooses the sym 4 function and the number of decomposing layers is 5 .

(2) The wavelet coefficients in all the scales are processed by soft threshold. For keeping the whole figure of signals constant, all the low frequency coefficients are preserved. We assume the threshold $\lambda=\sigma \sqrt{2 \log N}$, and every wavelet coefficient is processed by applying soft threshold.

$$
\widetilde{w}_{j, k}= \begin{cases}w_{j, k}-\lambda & w_{j, k} \geq \lambda \\ 0 & \left|w_{j, k}\right|<\lambda \\ w_{j, k}+\lambda & w_{j, k} \leq-\lambda\end{cases}
$$

In the practical calculation, all the variances of noises are unknown, which usually have to be estimated. Because the noises mainly focus on the most high resolution grade $J-1$, the standard deviations of noises are estimated by using wavelet coefficients.(Sun, Yankui. 2005)

(3) The processed wavelet coefficients are reconstructed making use of inverse wavelet transform to renew the effective signals, which is shown in figure 3.

Comparing figure 3 with figure 2, it is found that the wavelet transform not only restrain noises but also preserve the characteristic plug points reflecting the original signals, so it has very good denoising effects.

\section{Conclusions}

The wavelet transform has good resolution in both time domain and frequency domain, so it has more advantages in dealing with non-stationary signals. This paper applies the wavelet transform in the process of denoising MCG-signals and the results show that wavelet threshold denoising method not only eliminates the random noises of MCG-signals but also greatly preserve the original features of signals.

\section{References}

Chen, Lin. (2006). Noise cancellation techniques in magneto cardio graphy based on wavelet packet method. Chinese Journal of Low Temperature Physics, 2006,28(2):164 167.

$\mathrm{Hu}$, Rong. (2005). A mathematical morphological filtering approach to remove the noise of MCG-signals. Modern Scientific Instruments, 2005,2:54 55.

Jia, Mingtao \& Wang, Yongchen. (2003). Application of wavelet transformation in signal processing for vibrating platform. Journal of Shenyang Institute of Technology, 2003,22(3):53 55.

Li, Bicheng \& Luo, Jianshu. (2005). Wavelet analysis and its application. Beijing: Electronic Industry Press, 2005.

Sun, Yankui. (2005). Wavelet analysis and its application. Beijing: Mechanical Industry Press, 2005. 
Wang, Wei. (2008). Removal of the baseline drift in magneto cardiogram using the morphological filter. Modern Scientific Instruments, 2008,1:93 95.

Zhang, Yuanxiang. (2004). Analysis of noise cancellation techniques in magneto cardio graphy based on fourier transform method. Journal of Dalian College, 2004,3(3):95 96.

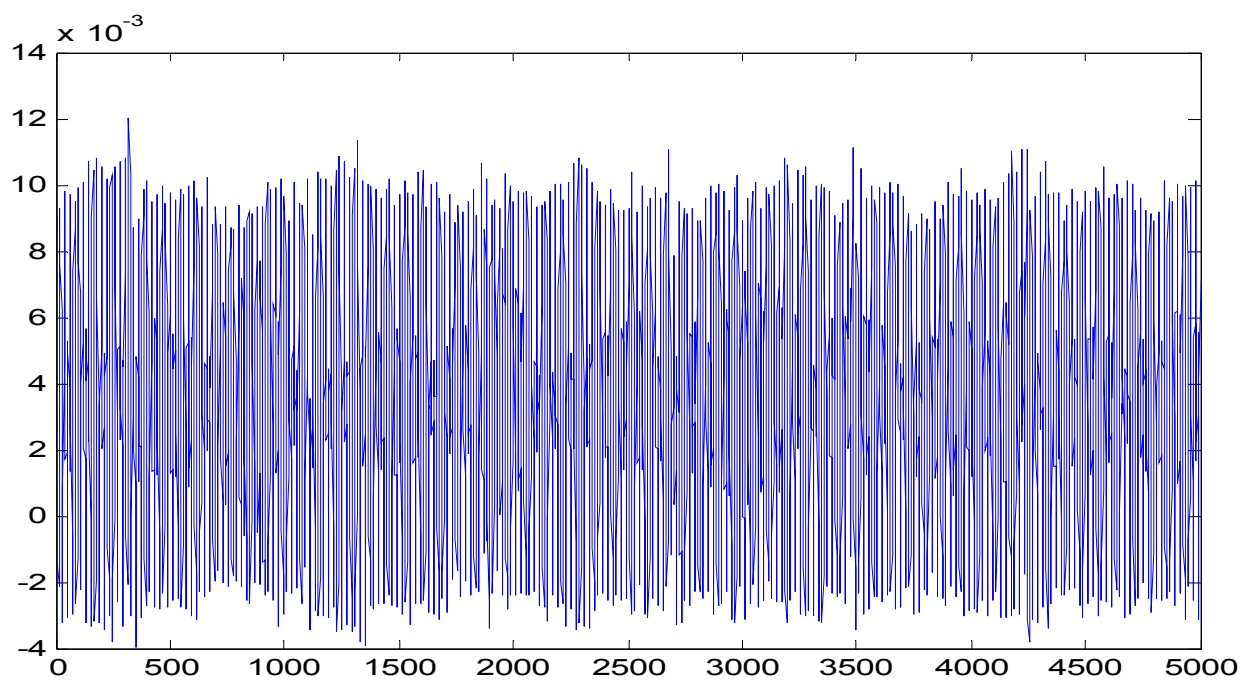

Figure 1. Signals surveyed originally

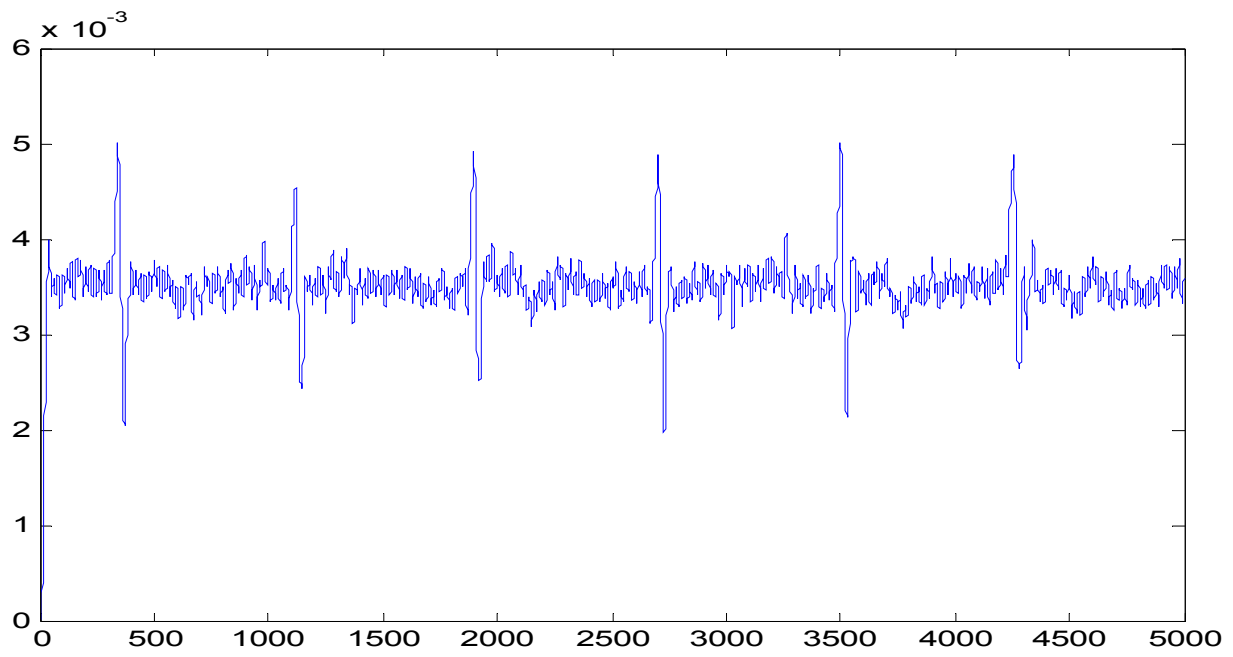

Figure 2. Signals eliminated power line interference 


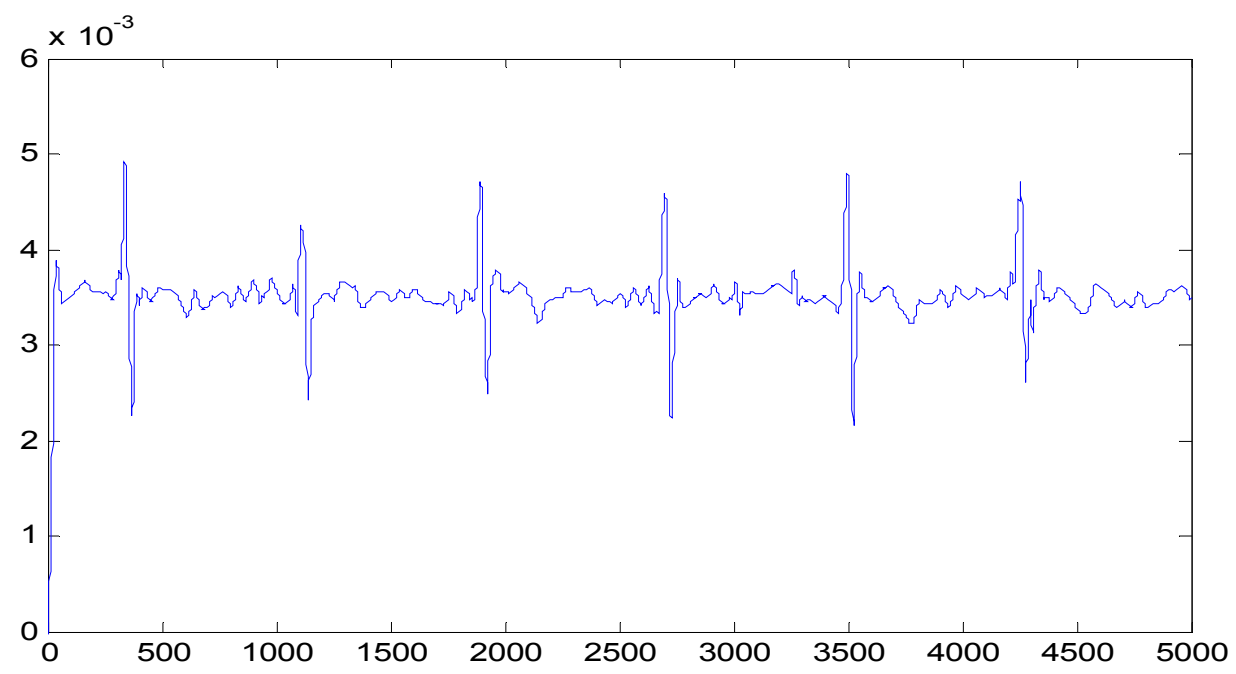

Figure 3. Signals through wavelet transformed and then filtered 